\title{
Ion acoustic instability of HPT particles, FAC density, anomalous resistivity and parallel electric field in the auroral region
}

\author{
C S JAyASREE ${ }^{1}$, G RENukA ${ }^{1}$ and C VEnugopal ${ }^{2}$ \\ ${ }^{1}$ Department of Physics, University of Kerala, Kariavattom, Trivandrum 695 581, India. \\ e-mail:drgrenuka@netscape.net \\ ${ }^{2}$ Department of Physics, University of Asmara, P.O.Box 1220, Eritrea.
}

\begin{abstract}
During the magnetic storm of 21st March 1990, the DE-1 spacecraft encountered the auroral region at high invariant latitude at altitudes ranging from a few thousand kilometers in the ionosphere to many earth radii in the magnetosphere. The magnetic field perturbations interpretable as field aligned current (FAC) layers and the electrostatic turbulence possibly due to electrostatic ion acoustic instability driven by these currents are shown. The critical drift velocity of Hot Plasma Torus (HPT) electrons and the growth rate of ion acoustic wave as a function of electron to ion temperature ratio $\left(T_{e} / T_{i}\right)$ for low and high current densities and energy of HPT electrons are found out. The intense FAC destabilizes the ion acoustic wave and the resultant electrostatic turbulence creates an anomalous resistivity. The current driven resistivity produces parallel electric field and high power dissipation. The anomalous resistivity $\eta$, potential difference along the auroral field lines $V_{\|}$, intensity of electric field turbulence $E_{\|}$and power produced per unit volume $P$ are computed. It is found that the change in westward magnetic perturbation increases $J_{\|}, \eta, V_{\|}, E_{\|}$and $P$. Hence HPT electrons are heated and accelerated due to power dissipation during magnetically active periods in the auroral region. Concerning, applications, such HPT electrons can be used in particle accelerators like electron ring accelerator, smokatron etc.
\end{abstract}

\section{Introduction}

Wave particle interaction theory has revealed that when the plasma electrons drift with respect to ions with an average speed larger than some threshold value, both the electrostatic and the electromagnetic field grow at the expense of the electron kinetic energy and as a result, various instabilities set in. The magnetometer data indicate that these waves are spatially correlated with perturbations; we interpret them as field aligned current (FAC) layers directed into and out of the auroral ionosphere. Kinetic theory describes a variety of instabilities like Buneman, ion acoustic and ion cyclotron etc., that are driven by FACs. Hence, for the first time, we study the electrostatic ion acoustic instability of HPT particles. HPT is defined as hot plasma particles with average energy of $1-10 \mathrm{keV}$ having several degrees of latitude in width in the magnetosphere.

FACs predicted by Birkeland (1908) were observed by TRIAD (Zmuda and Armstrong 1974) and Iijima and Potemra (1976) after detailed study of FAC, separated them into region 1 and region 2 current systems. It is proposed that the region $1 /$ region 2 FACs can be generated as a result of natural distortion of the HPT due to the solar wind convection (Yamamoto et al 1996). Whistler mode instability is produced due to FACs and parallel electric field in the earth's magnetosphere (Maru et al 1999). Ion demagnetization in the plasma

Keywords. HPT; FACs; anomalous resistivity; parallel electric field.

Proc. Indian Acad. Sci. (Earth Planet. Sci.), 112, No. 4, December 2003, pp. 559-567

(C) Printed in India. 
sheet caused the formation of FAC that can trigger a magnetosphere-ionosphere coupling feedback instability which may play an important role in substorm and auroral arc generation (Lyatsky and Hamza 2001).

In this paper, using the high time resolution magnetometer data (MAG-A) from the Dynamic Explorer (DE-1), the value of FAC density is estimated. The growth rate of the ion acoustic wave, anomalous resistivity, potential difference along the auroral field lines, electric field intensity of turbulence and power produced per unit volume in the auroral latitude during a geomagnetic storm, are also computed.

\section{Data and method}

\subsection{Field aligned currents}

To compute the value of FAC density, 6 seconds average magnetic field data of DE-1 is used. The spacecraft was launched on 3rd August 1981 into coplanar polar orbits at an altitude range 570 to $23000 \mathrm{~km}$ for studying interactive processes within the atmosphere-ionosphere-magnetosphere system. The MAG-A high time resolution data set consists of triaxial fluxgate measurements taken every $62.5 \mathrm{msec}$ (i.e., 16 vectors/second). The gradual commencement storm of March 21st 1990 (UT 0001-2000) has been considered and it is found that the $B_{y}$ perturbation is negative or westward throughout the disturbance region of about few thousand kilometers and it lies within $63^{\circ} \leq \wedge \leq$ $76^{\circ}$. The electrons and protons are represented as HPT electrons and protons throughout this paper.

The density and flow direction of equivalent FACs are determined from the magnetic disturbance $B_{y}$ using (Potemra 1985),

$$
J_{\|}=\frac{1}{\mu_{0}} \frac{\partial B_{y}}{\partial x}=8 \times 10^{-4} \frac{\partial B_{y}}{\partial x} \mathrm{~A} / \mathrm{m}^{2},
$$

where altitude $x$ is in meters, $\mu_{0}=4 \pi \times 10^{-7} \mathrm{H} / \mathrm{m}$ is the permeability of free space, $B_{y}$ is in nanoteslas (nT) and is directed towards north.

\subsection{Ion acoustic instability}

The electron current driven ion acoustic instability is that the combined equilibrium distribution function consisting of the drifting hot HPT electron background and the cold ions, exhibits a positive slope where resonance between waves and HPT particles can occur. We can use the linear dispersion relation of ion acoustic waves as (Galeev and Sagdeev 1984)

$$
\begin{aligned}
\varepsilon(\omega, k) & \equiv 1-\frac{\omega_{p i}^{2}}{\omega^{2}} \\
+\frac{\omega_{P i}^{2}}{k^{2} c_{s}^{2}} & {\left[1+i \sqrt{\frac{\pi m_{e}}{2 m_{i}}}\left(\frac{\omega}{k c_{s}}-\frac{V_{d}}{c_{s}} \cos \theta\right)\right], }
\end{aligned}
$$

where

$$
\begin{aligned}
& V_{d}=-\frac{J_{\|}}{e n_{0}}, \\
& c_{s}=V_{e}\left(\frac{m_{e}}{m_{i}}\right)^{1 / 2},
\end{aligned}
$$

the electron distribution function is assumed to be Maxwellian shifted by the drift velocity $V_{d}, \omega_{p i}$ is the ion plasma frequency, $\theta$ is the angle between the wave vector $k$ and the direction of the current, $e$ is the electronic charge, $n_{0}$ is the number density of HPT electrons and $n_{0}$ is taken as $10^{6} / \mathrm{m}^{3}$ (Yamamoto et al 1996), $c_{s}$ is the ion acoustic velocity, $V_{e}$ is the thermal velocity of electrons and $m_{e}$ and $m_{i}$ are the masses of electrons and ions respectively.

The basic requirement for ion acoustic current driven instability is a cold ion and hot electron plasma with drift velocity exceeding the ion acoustic velocity $\left(V_{d}>c_{s}\right)$ and we also assume that $V_{e} \gg V_{d}$. The dominant range of ion acoustic instability is therefore realised when $T_{e} \gg T_{i}$, the wavelength is much larger than the Debye wavelength for HPT electrons $\lambda_{D}, k^{2} \lambda_{D}^{2} \ll 1$ and $V_{d}>\frac{\omega}{k} \approx c_{s}$. Under these conditions ion Landau damping is unimportant and the growth rate $\gamma_{i a}$ (Treumann and Baumjohann 1997) is

$$
\gamma_{i a}=\left(\frac{\pi m_{e}}{8 m_{i}}\right)^{1 / 2} \frac{\omega_{i a}}{\left(1+k^{2} \lambda_{D}^{2}\right)^{3 / 2}}\left(\frac{k V_{d}}{\omega_{i a}}-1\right),
$$

where

$$
\omega_{i a}^{2}=\frac{\omega_{p i}^{2}}{\left(1+\frac{1}{k^{2} \lambda_{D}^{2}}\right)}\left[1+\frac{3 T_{i}}{T_{e}}\left(1+k^{2} \lambda_{D}^{2}\right)\right],
$$

$\omega_{i a}$ is the ion acoustic wave frequency, $T_{e}$ and $T_{i}$ are the electron and ion temperatures respectively. The critical drift velocity $V_{c r}$ can be expressed as (Papadopoulos 1977)

$$
\begin{array}{r}
V_{c r}=\frac{\left(\frac{T_{e}}{m_{i}}\right)^{1 / 2}}{\left(1+k^{2} \lambda_{D}^{2}\right)^{1 / 2}}\left[1+\left(\frac{T_{e}}{T_{i}}\right)^{3 / 2}\left(\frac{m_{i}}{m_{e}}\right)^{1 / 2}\right. \\
\left.\exp \left(\frac{-3}{2}-\frac{\left(\frac{T_{e}}{T_{i}}\right)}{2\left(1+k^{2} \lambda_{D}^{2}\right)}\right)\right] \cdot(7)
\end{array}
$$




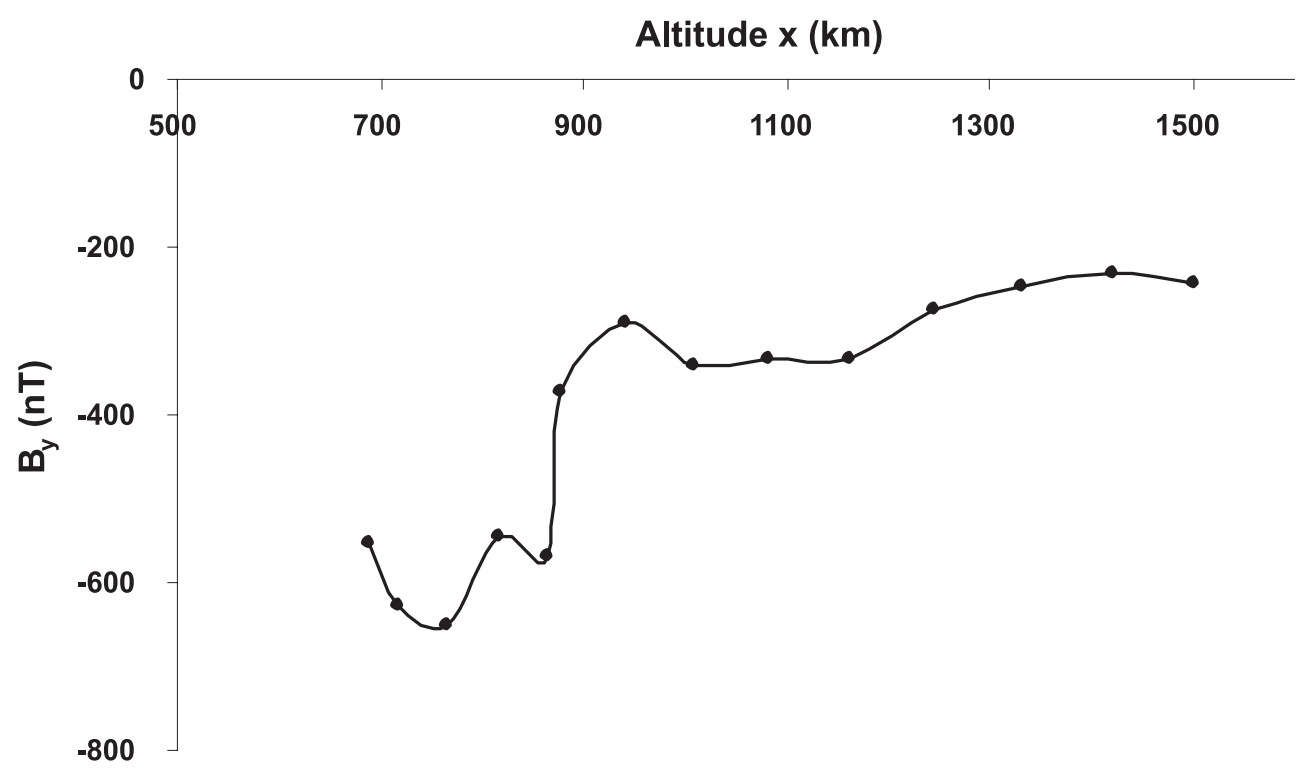

Figure 1. The variation of magnetic perturbation $B_{y}$ with altitude $x$.

\subsection{Anomalous resistivity and field aligned potential drop}

When instability occurs the field energy grows exponentially hence the loss of kinetic energy of electrons suddenly increases and the current carried by these electrons is suddenly disrupted. Such instabilities can produce anomalous resistivity. Swift (1965) proposed the existence of the ion acoustic instability in the auroral plasma which could lead to anomalous resistivity, a field aligned electric potential and the acceleration of auroral electrons. The ion acoustic anomalous resistivity $\eta$ is estimated using the relation (Treumann and Baumjohann 1997)

$$
\eta=\frac{\nu_{i c}}{\omega_{p e}^{2}} \frac{\varepsilon_{0}}{2}
$$

where

$$
\nu_{i c}=0.01 \omega_{p i}\left(\frac{V_{d}}{c_{s}}\right)\left(\frac{T_{e}}{T_{i}}\right),
$$

$\nu_{i c}$ is the ion acoustic collision frequency, $\omega_{p e}$ is the electron plasma frequency and $\varepsilon_{0}$ is the dielectric constant in free space. The Ogo 5 and Ov33 measurements may indicate that FAC regions and electrostatic turbulence associated with them can produce anomalous resistivity on high latitude auroral regions on the night side (Scarf et al 1973). The extent of plasma wave turbulence $l$ is about $2 R_{E}\left(1 R_{E}=6371 \mathrm{~km}\right.$, the radius of the earth) of the current path (Fredricks and Scarf 1973). Then the field aligned potential drop $V_{\|}$along the auroral field lines is

$$
V_{\|}=J_{\| \eta} l
$$

\subsection{Parallel electric field and power dissipation}

The ion acoustic wave instability might produce large resistivity which could create a very large electric field parallel to the magnetic field. Intensity of electric field turbulence $E_{\|}$is given by Mozer (1976)

$$
E_{\|}=J_{\|} \eta
$$

The current driven resistivity produces high local power dissipation which leads to excessive plasma heating in the topside ionosphere or low magnetosphere. Anomalously high values of $\eta$ means high power dissipation and power produced per unit volume $P$ is given by Papadopoulos and Coffey (1974)

$$
P=\eta J_{\|}^{2}
$$

\section{Numerical results and discussion}

Figure 1 shows the variation of the magnetic perturbation $B_{y}$ with altitude $x$. These disturbances are observed during the disturbed conditions $\left(4^{+}=\right.$ $K_{p} \leq 6^{-}$) of March 21st 1990 in the early and late afternoon sector (1400-2000 MLT) at high invariant latitude $63^{\circ} \leq \wedge \leq 76^{\circ}$ auroral field lines at altitudes ranging from $600-1500 \mathrm{~km}$. It is found that magnetic perturbations are negative or westward throughout the disturbance region. Satellite observations of magnetic perturbations transverse to the main geomagnetic field have provided the most direct evidence for the existence of FACs. The figure clearly depicts that as the altitude increases there is a decrease in the variation of magnetic field strength $B_{y}$ due to the geomagnetic storm. 


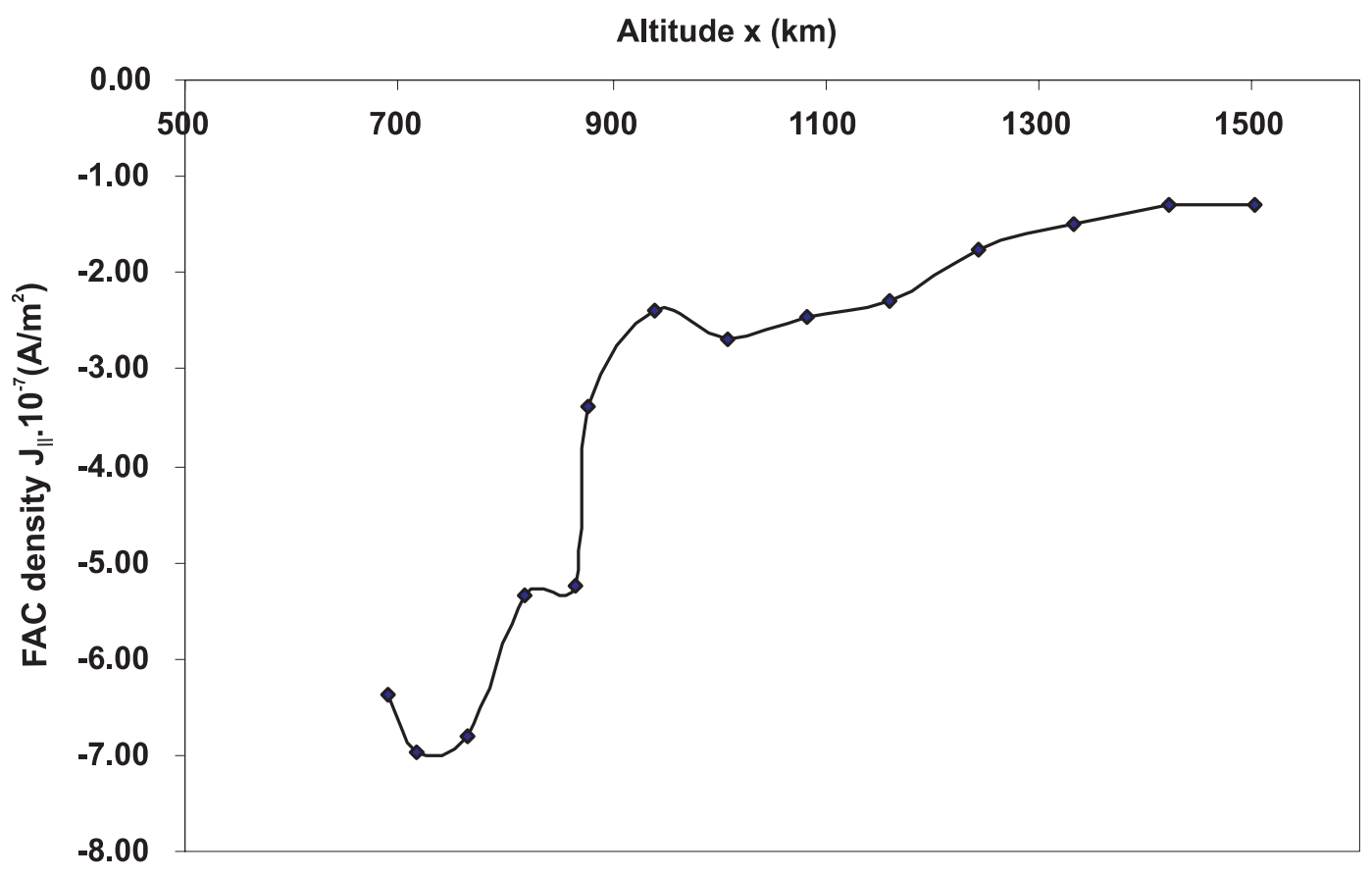

Figure 2. The FAC density $J_{\|}$is plotted against altitude $x$.

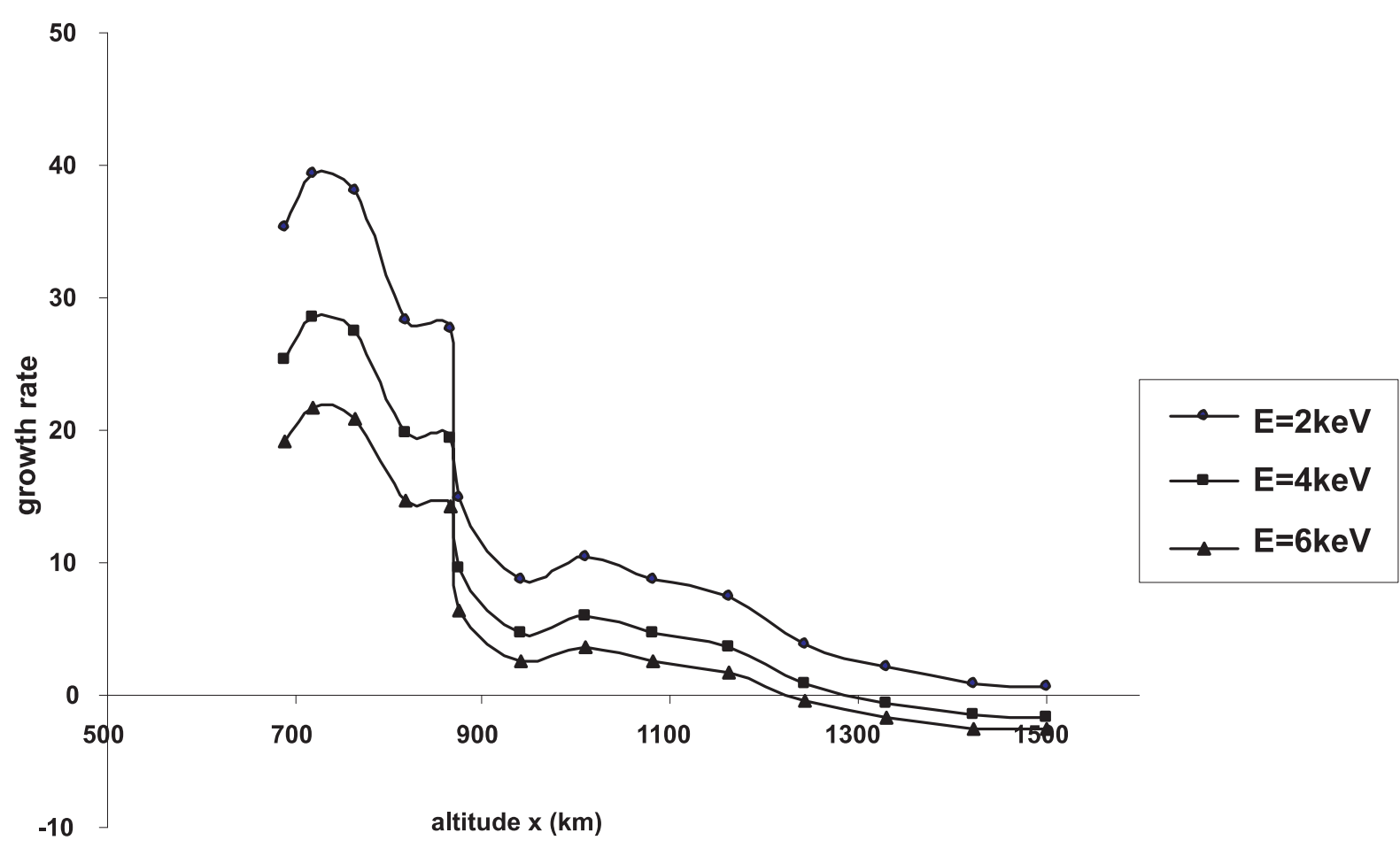

Figure 3. Growth rate $\gamma_{i a}$ as a function of altitude $x$ for different energy $E=2,4,6 \mathrm{keV}$.

Figure 2 represents the nature of variation of FAC density $J_{\|}$with $x$. We compute the FAC density from the $B_{y}$ variations using equation (1) and negative values denote the FAC flow is upward in the equatorward part and downward in the poleward part. The current density lies between $-1.29 \times 10^{-7} \mathrm{~A} / \mathrm{m}^{2}$ and $-6.98 \times 10^{-7} \mathrm{~A} / \mathrm{m}^{2}$ consistent with the results of Zmuda and Armstrong
(1974) and Iijima and Potemra (1976). It is clear from the figure that $J_{\|}$increases with decreasing altitude as the field strength increases (Fredricks and Scarf 1973). The threshold for excitation of ion-acoustic wave is high and requires high velocities of the current carriers to exceed the ionacoustic velocity and is thus strong FACs. The critical current required to excite this wave is 

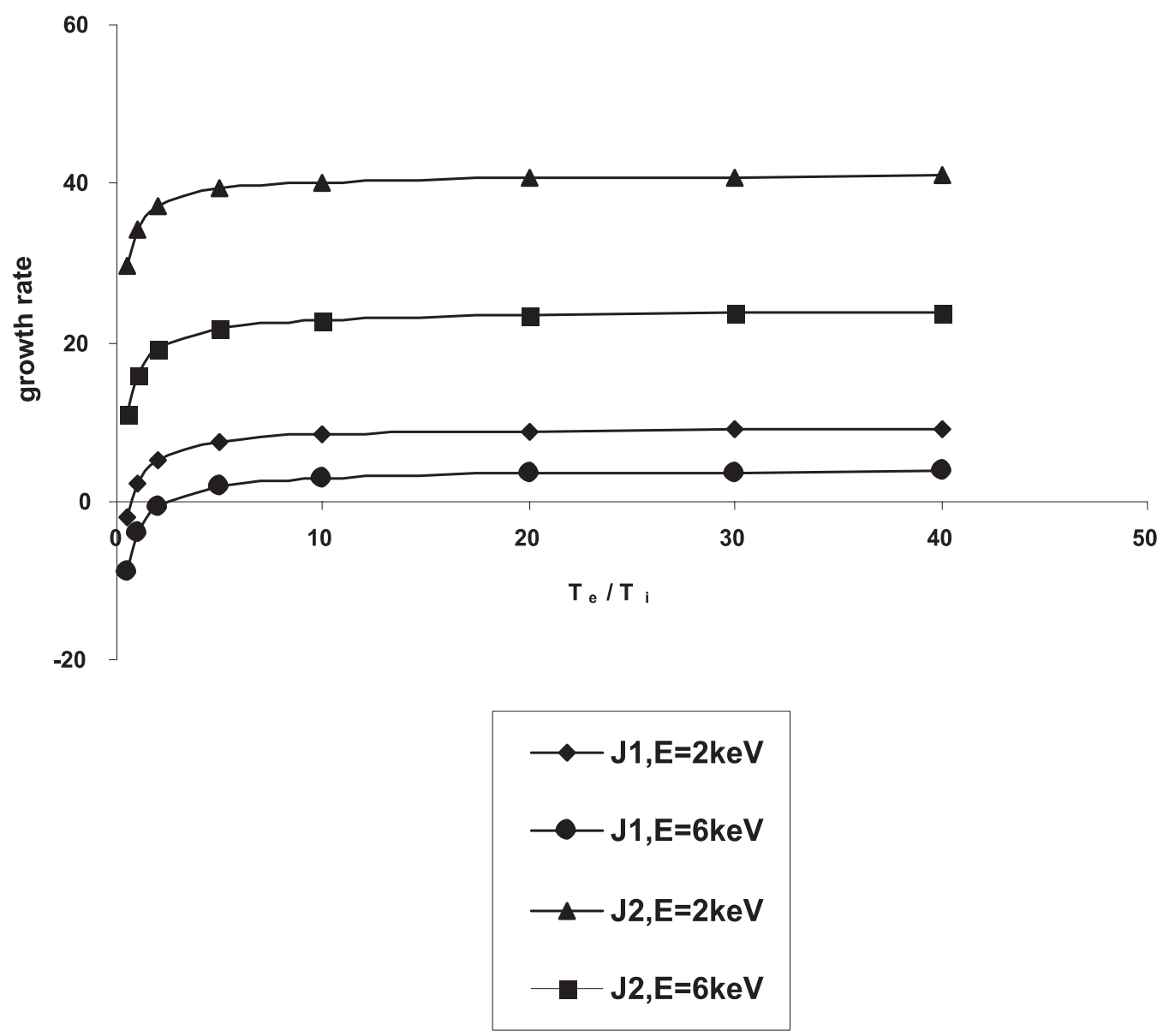

Figure 4. The variation of growth rate with $T_{e} / T_{i}$ for different FAC density $J_{1}$ and $J_{2}$ and energy $E=2,6 \mathrm{keV}$.

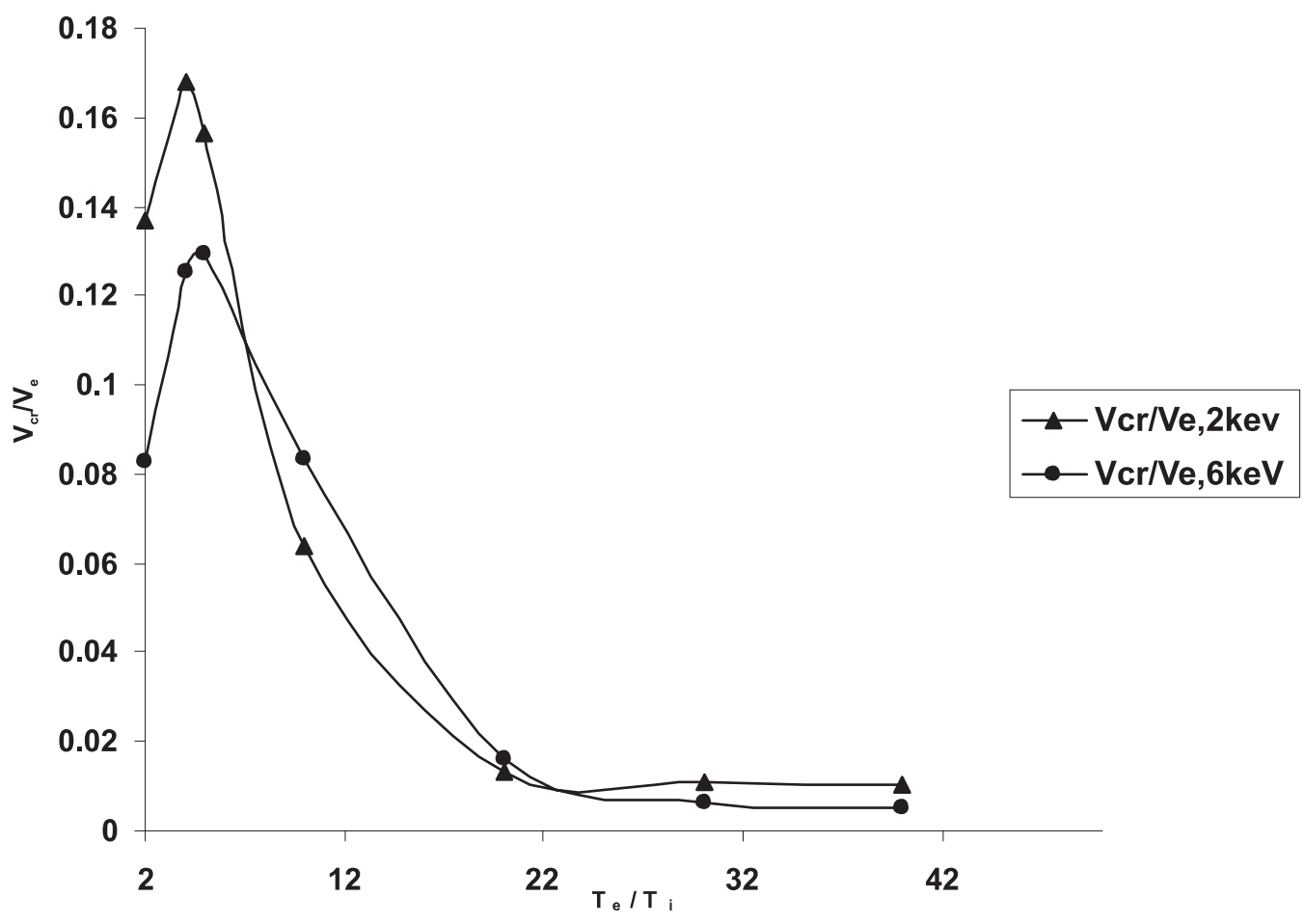

Figure 5. The plot of $V_{c r} / V_{e}$ versus $T_{e} / T_{i}$ for different energy $E=2,6 \mathrm{keV}$. 
greater than $-1.70 \times 10^{-7} \mathrm{~A} / \mathrm{m}^{2}$. In this paper, we select $J_{1}=-2.29 \times 10^{-7} \mathrm{~A} / \mathrm{m}^{2}$ as the minimum value and $J_{2}=-6.98 \times 10^{-7} \mathrm{~A} / \mathrm{m}^{2}$ as the maximum value. These maximum and minimum values of FAC density $J_{\|}$and energy $E=2,6 \mathrm{keV}$ are used in the following sections.

Figure 3 shows the nature of variation of $\gamma_{i a}$ as a function of altitude $x$ for different values of energy $E=2,4,6 \mathrm{keV}$. The growth rate is estimated by using equation (5) and the various parameters used as $\bar{\omega}=1000 \mathrm{rad} / \mathrm{sec}$ and $k=10^{-3} / \mathrm{m}$ (Gurnett and Frank 1977). Hence wave phase velocity, $\bar{\omega} / k=10^{6} \mathrm{~m} / \mathrm{sec}$ which is less than the electron drift velocity. It is found that $\gamma_{i a}$ increases with decreasing $x$ as $J_{\|}$increases and as $E$ of the electron increases $\gamma_{i a}$ decreases. This is because, as $E$ increases, $k$ becomes shorter than $\lambda_{D}$, hence $k^{2} \lambda_{D}^{2} \geq 1$ and $V_{d} \leq c_{s}$. Under these conditions ion Landau damping is important and as a result $\gamma_{i a}<0$ i.e., the instability becomes very weak (Kindel and Kennel 1971).

Figure 4 indicates the variations of $\gamma_{i a}$ with electron to ion temperature ratio $T_{e} / T_{i}$ for $J_{1}$ and $J_{2}$ and $E=2,6 \mathrm{keV}$. In ion-acoustic wave, electron growth is balanced with ion Landau damping.
When $T_{e} / T_{i} \leq 1, \gamma_{i a}<0$, i.e., the instability is weak. In this case, the ion temperature is large and the wave phase velocity becomes less than the background ion thermal velocity. So the ion contribution to the growth rate corresponds to Landau damping of the waves. When $T_{e} / T_{i}=1$, the phase velocity exceeds the ion thermal speed, Landau damping is weak and the drift must exceed the ion thermal speed for instability. It is seen that for $T_{e} / T_{i}>5, \gamma_{i a}$ becomes almost constant which determines the saturation level of ion acoustic waves.

The critical relative drift velocity $V_{c r} / V_{e}$ as a function of $T_{e} / T_{i}$ for $E=2,6 \mathrm{keV}$ is shown in figure $5 . V_{c r} / V_{e}$ is computed by using equation (7) at $T_{e} / T_{i}=0.5,1,2,5,10-40$. When $T_{e} \sim T_{i}$, the phase velocity of the ion acoustic wave approaches the ion thermal speed $V_{i}$ and hence heavy ion Landau damping results. This makes the ion acoustic wave difficult to excite and results in an increase in the electron threshold drift speed to $V_{e}$. For high $T_{e} / T_{i}$, the threshold gets low and is just the ion acoustic speed due to weak ion Landau damping. When the current is weak and $T_{e} \sim T_{i}$, the ion cyclotron instability may be excited. When the current is increased, the growth rate becomes larger than the
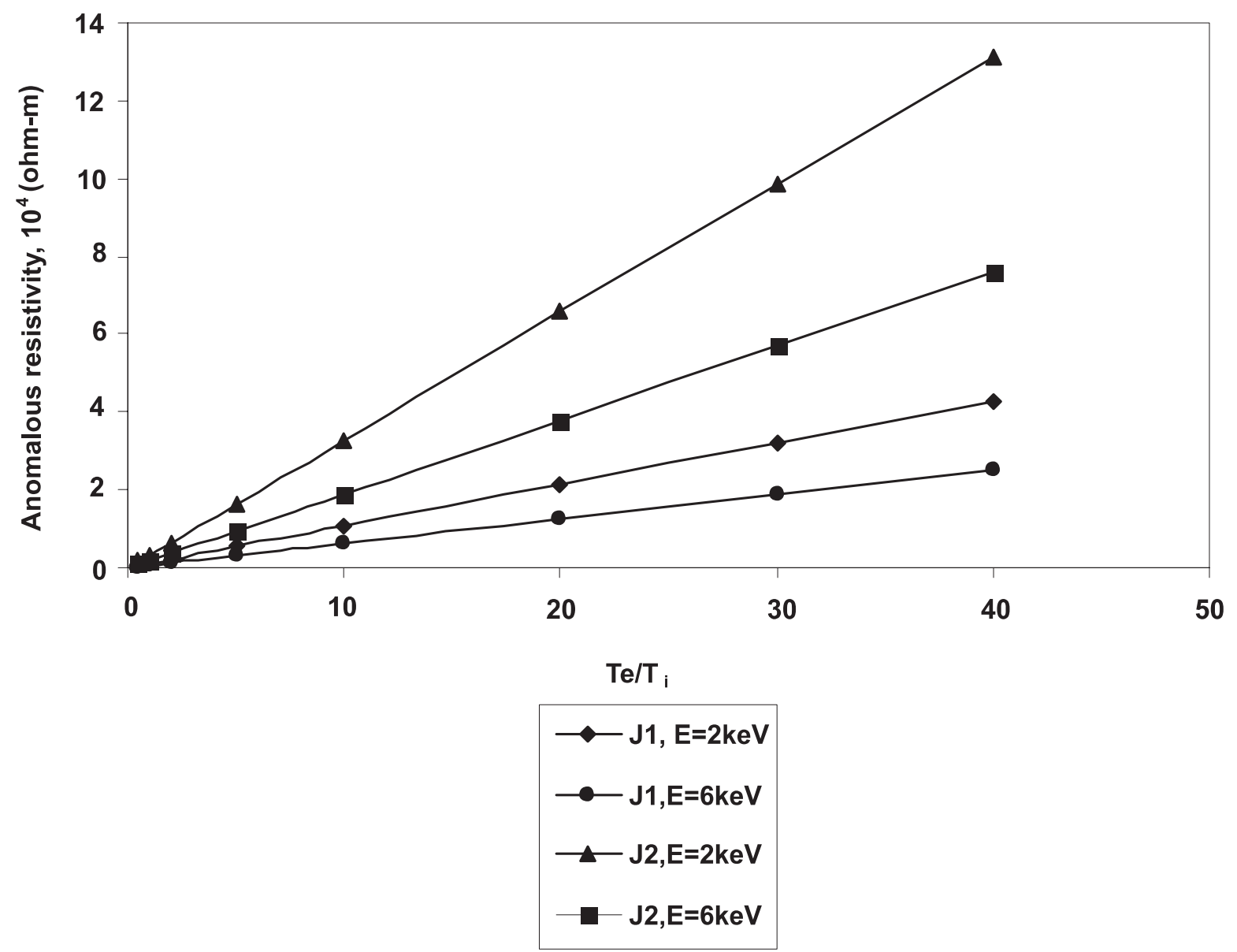

Figure 6. The variation of anomalous resistivity $\eta$ with $T_{e} / T_{i}$ for different FAC density $J_{1}$ and $J_{2}$ and energy $E=2,6 \mathrm{keV}$. 
ion cyclotron frequency, then the periodic gyro motion of the ions is destroyed and the ions become demagnetized. When this happens, the ion cyclotron wave is lost and the instability changes into ion acoustic type. If the current is further increased, $V_{d}>V_{e}$, the electron-ion two stream (Buneman) instability is excited.

The variation of the anomalous resistivity $\eta$ with $T_{e} / T_{i}$ for $J_{1}$ and $J_{2}$ and $E=2,6 \mathrm{keV}$ can be seen in figure 6 . The ion-acoustic collision frequency is estimated by using equation (9) and then $\eta$ by equation (8). For high temperature ratio, $\eta$ increases with increasing $J_{\|}$and decreases as the energy of the HPT electron increases. The $\eta$ on auroral field lines is a factor of $10^{2}$ to $2 \times 10^{4}$ greater than Spitzer value (Mozer 1976). As the FAC density increases from a threshold value, instability sets in; this may cause an anomalously large resistivity (Hasegawa 1975). It is clear from the figure that $\eta$ is very low for weak current $J_{1}$ and high for strong current $J_{2}$.

In figure 7 we plot the variation of potential difference $V_{\|}$with $T_{e} / T_{i}$ for $J_{1}$ and $J_{2}$ at $E=2,6 \mathrm{keV}$.
Using equation (10) we compute the value of $V_{\|}$ and it depends on the values of $\eta$ and $J_{\|}$. As the current and resistivity increases, $V_{\|}$also increases leading to particle acceleration and auroral arc formation (Fredricks and Scarf 1973).

Figure 8 shows the variation of parallel electric field $E_{\|}$with $T_{e} / T_{i}$ for $J_{1}$ and $J_{2}$ at $E=2,6 \mathrm{keV}$. $E_{\|}$is calculated by using equation (11). $E_{\|}$also increases with $T_{e} / T_{i}$ for increasing $J_{\|}$and decreasing energy. From this we can infer that, ion acoustic instability tends to block the current by dissipating the current energy into the electric field energy, thus producing turbulence of electric field strengths up to $100 \mathrm{mV} / \mathrm{m}$ in the auroral region. This is in agreement with the observation of electrostatic turbulence measured from Ogo5 (Fredricks and Scarf 1973).

Figure 9 indicates the variation of power produced per unit volume $P$ with $T_{e} / T_{i}$ for $J_{1}$ and $J_{2}$ and $E=2,6 \mathrm{keV}$. The value of $P$ is computed by using equation (12) and the nature of variation is the same as that of $\eta, V_{\|}$and $E_{\|}$. It is found that anomalously high values of $\eta$ means anomalously

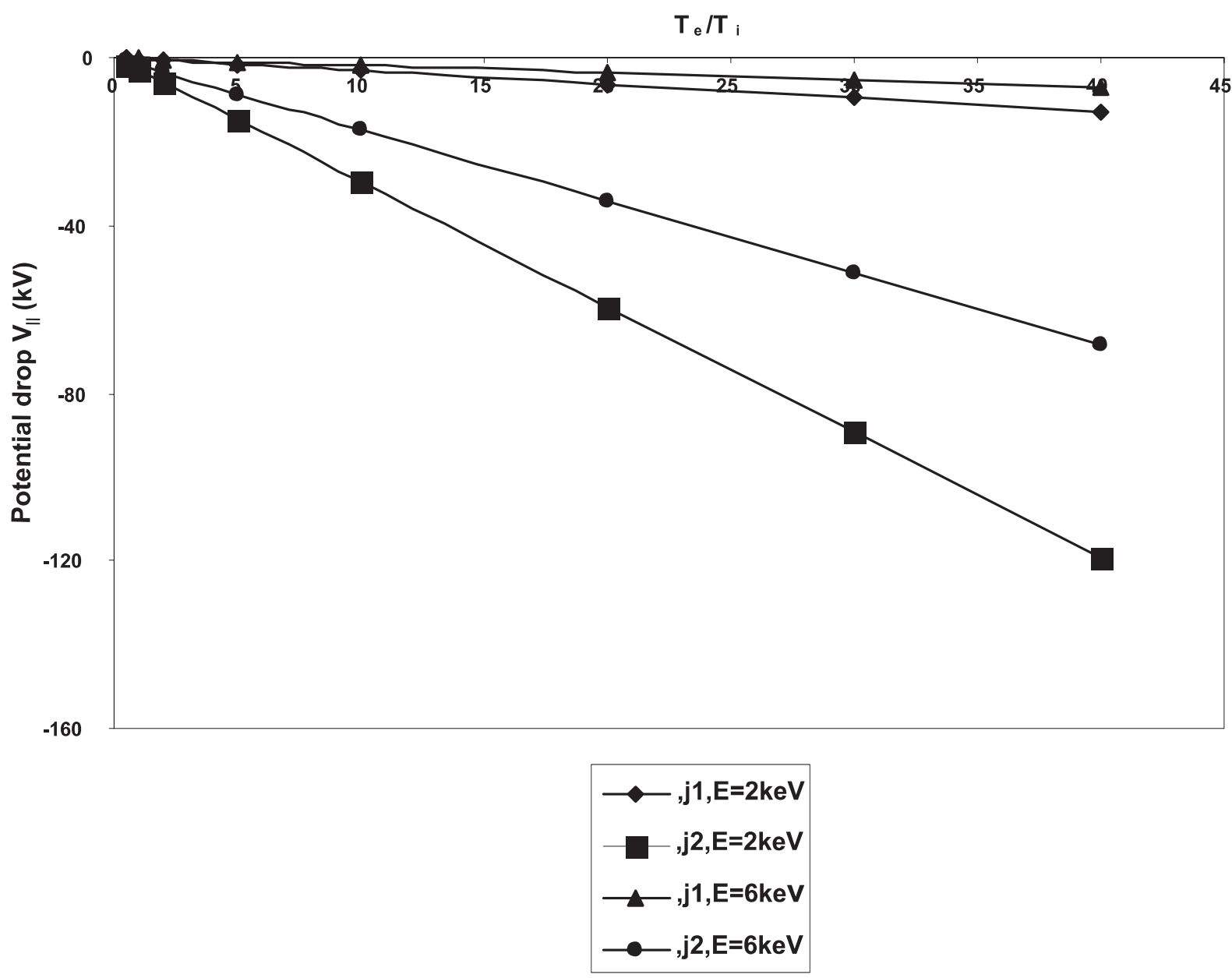

Figure 7. Field aligned potential difference $V_{\|}$is plotted against $T_{e} / T_{i}$ for different FAC density $J_{1}$ and $J_{2}$ and energy $E=2,6 \mathrm{keV}$. 


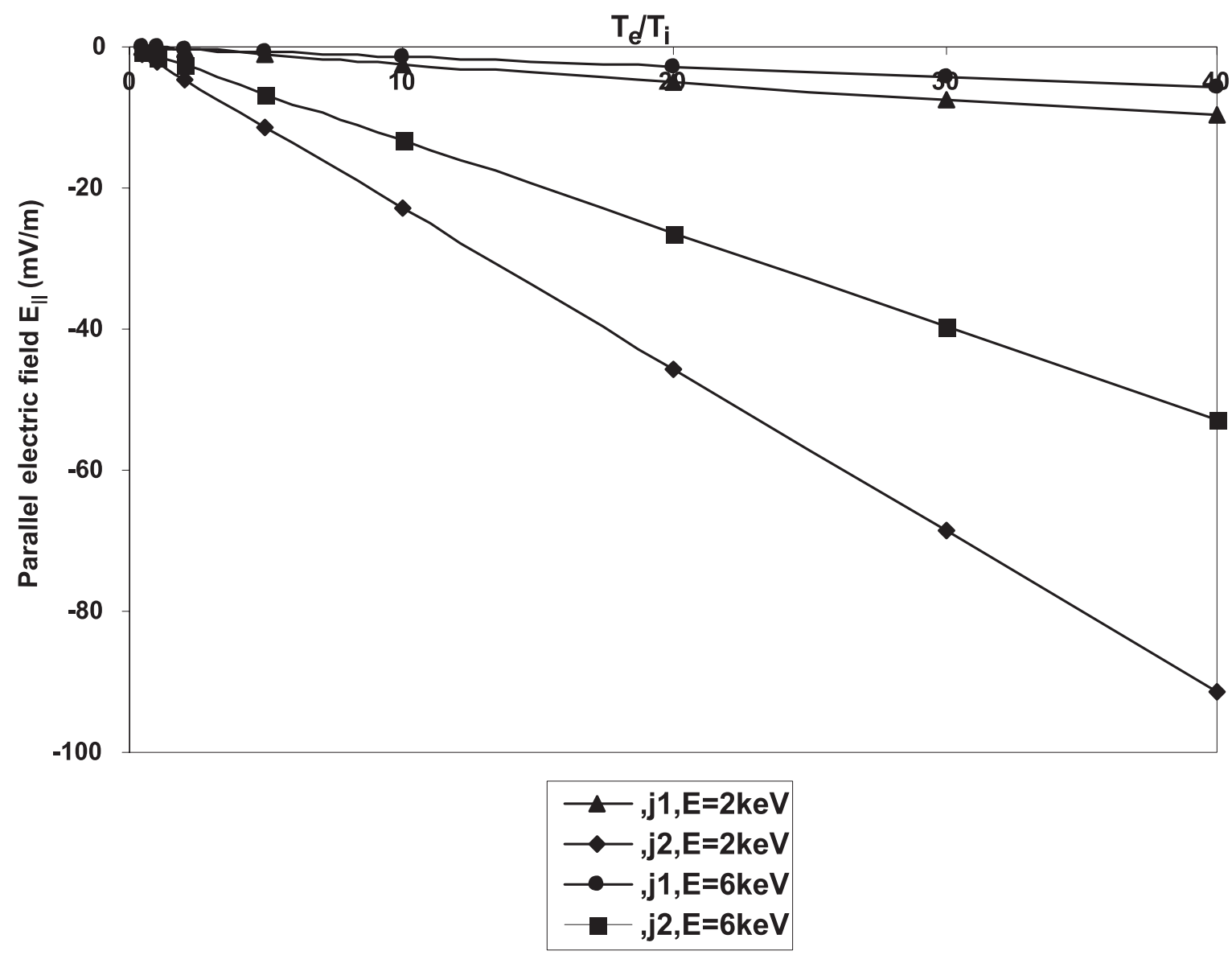

Figure 8. The parallel electric field $E_{||}$as a function of $T_{e} / T_{i}$ for different FAC density $J_{1}$ and $J_{2}$ and energy $E=2,6 \mathrm{keV}$.

high power dissipation. A current density of $-6.98 \times 10^{-7} \mathrm{~A} / \mathrm{m}^{2}$ produces an $E_{\|}$of $-11.45 \mathrm{mV} / \mathrm{m}$ and dissipates a power of $7.99 \times$ $10^{-9} \mathrm{~W} / \mathrm{m}^{3}$. Mozer (1976) computed a current density of $3 \times 10^{-5} \mathrm{~A} / \mathrm{m}^{2}$ produces an $E_{\|}$of $10 \mathrm{mV} / \mathrm{m}$ dissipates a power of $3 \times 10^{-7} \mathrm{~W} / \mathrm{m}^{3}$. So this high power dissipation leads to excessive plasma heating in the topside ionosphere or low magnetosphere.

\section{Conclusion}

The existence of a large FAC during auroral substorm activities has led to the speculation that this current may generate an ion acoustic instability which may cause an anomalously large resistivity which could create a very large electric field parallel to the magnetic field. The electric field turbulence produced by this instability grows to large amplitude and the associated anomalous resistivity produces a large potential difference of the order of kilovolts along the magnetic field in auroral regions. Since FACs, anomalous resistivity, potential drop along the auroral field lines, intensity of electric field turbulence and power produced per unit volume are playing important roles in ion acoustic instability, a clear picture of this instability in the auroral region associated with BEN due to turbulence can be obtained.

\section{References}

Birkeland K 1908 On the cause of magnetic storms and the origin of terrestrial magnetism; In: The Norwegian Aurora Polaris expedition 1902-03 (ed) H Aschehoug, vol. I (Oslo, Norway)

Fredricks R W and Scarf F L 1973 Field aligned currents, plasma waves and anomalous resistivity in the disturbed polar cusp; J. Geophys. Res. 78 2133-2141

Galeev A A and Sagdeev R Z 1984 Current instabilities and anomalous resistivity of plasma; In: Hand book of plasma physics (eds) A A Galeev and R N Sudan, Vol. II (Amsterdam, North Holland) pp 271-303

Gurnett D A and Frank L A 1977 A region of intense plasma wave turbulence on auroral field lines; J. Geophys. Res. 82 1031-1050

Hasegawa A 1975 Plasma instabilities and nonlinear effects; In: Physics and Chemistry in space (eds) J G Roederer and J T Wasson Vol. VIII (NewYork: Springer) pp 62

Iijima T and Potemra T A 1976 The amplitude distribution of field aligned currents at northern high latitudes observed by TRIAD; J. Geophys. Res. 81 2165-2174 


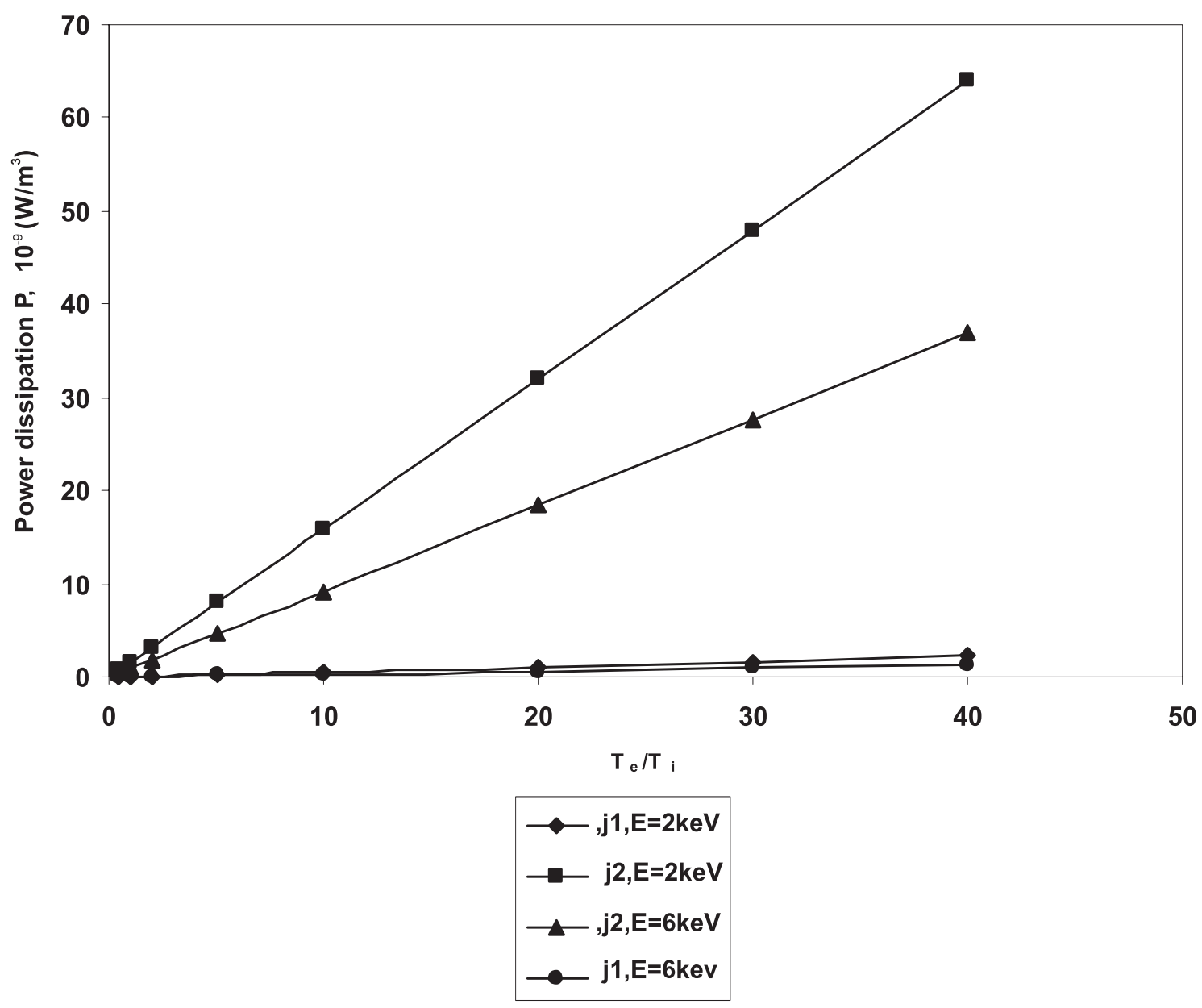

Figure 9. Power dissipation per unit volume $P$ is plotted against $T_{e} / T_{i}$ for different FAC density $J_{1}$ and $J_{2}$ and energy $E=2,6 \mathrm{keV}$.

Kindel J M and Kennel C F 1971 Topside current instabilities; J. Geophys. Res. 76 3055-3078

Lyatsky W and Hamza A M 2001 Possible role of ion demagnetization in the plasma sheet in auroral arc and substorm generation; Space Sci. Rev. 95 373-85

Maru, Harsha, Kumar, Sushil and Gwal A K 1999 Role of field aligned currents and parallel electric field on the generation of whistler mode instability in the earth's magnetosphere; Indian J. Radio Space Phys. 28 95-102

Mozer F S 1976 Anomalous resistivity and parallel electric fields; In: Magnetospheric particles and fields (ed) B M McCormac (Dordrecht, Holland: D. Reidel) pp $125-136$

Papadopoulos K 1977 A review of anomalous resistivity for the ionosphere; Rev. Geophys. and Space Physics 15 113127

Papadopoulos K and Coffey T 1974 Anomalous resistivity in the auroral plasma; J. Geophys. Res. 79 1558-1561
Potemra T A 1985 Field aligned currents; Space Sci. Rev. 42 295-311

Scarf F L, Fredricks R W, Russell C T, Kivelson M, Weugebauer M and Chappel C R 1973 Observation of a current driven plasma instability at the outer zone plasma sheet boundary; J. Geophys. Res. 78 2150-2165

Swift D W 1965 A mechanism for energizing electrons in the magnetosphere; J. Geophys. Res. 70 3061-3073

Treumann R A and Baumjohann W 1997 Electrostatic instabilities; In: Advanced Space Plasma Physics (London: Imperial College Press) pp 69-102

Yamamoto T, Inoue S, Nishitani N, Ozaki M and Meng C I 1996 A theory for generation of the paired region 1 and region 2 field aligned currents; J. Geophys. Res. 101 27199-27222

Zmuda A J and Armstrong J C 1974 The diurnal flow pattern of field aligned currents; J. Geophys. Res. 79 $461-469$ 